Tropical Journal of Pharmaceutical Research October 2014; 13 (10): 1621-1627

ISSN: $1596-5996$ (print); 1596-9827 (electronic)

(c) Pharmacotherapy Group, Faculty of Pharmacy, University of Benin, Benin City, 300001 Nigeria.

All rights reserved.

Available online at http://www.tjpr.org

Original Research Article

http://dx.doi.org/10.4314/tjpr.v13i10.8

\title{
Inhibition of Dengue Virus 3 in Mammalian Cell Culture by Synthetic Small Interfering RNAs Targeting Highly Conserved Sequences
}

\author{
Ummar Raheel* and Najam Us Sahar Sadaf Zaidi \\ Atta-ur-Rahman School of Applied Biosciences, National University of Sciences and Technology (NUST), H-12 Sector, \\ Islamabad, Pakistan
}

${ }^{\star}$ For correspondence: Email: ummarraheel@gmail.com; zaidi.sahar@gmail.com; Tel: +923325416727

Received: 28 June 2014

Revised accepted: 13 September 2014

\begin{abstract}
Purpose: To evaluate the inhibition of Dengue virus 3 by synthetic siRNAs targeting the untranslated regions UTR and structural regions of DENV3 genome in Vero-81 cell line.

Methods: Vero-81 cells transfected with synthetic siRNAs were challenged by DENV3. The effectiveness of siRNAs was confirmed by four established virus quantification procedures. Starting with focus assay, DENV3 was quantified using anti-E antibody (Envelope), in which DENV3 was quantified by counting the number of foci per well. Initial results were then confirmed by immuno-florescence assay (IFA) as the number of Vero-81 cells displaying DENV3 (Envelope) E antigen had a higher florescent intensity in comparison to cells lacking DENV3 replication. DENV3 RNA copy numbers were quantified by real-time quantative polymerase chain reaction RT-qPCR and in the final step supernatant of Vero-81 cells challenged with DENV3 was collected and protein analysis was performed to determine the presence of DENV3 E protein via western blot analysis

Results: A marked decrease in virus titer of DENV3 in Vero cells was observed with DV3UTR3'siRNA2 targeting the 3'UTR. Focus assay data revealed more than $70 \%$ reduction in DENV3 in Vero-81 cells treated with DV3UTR3'siRNA2. Images showing IFA of infected Vero-81 cells exhibited a major drop in DENV3 titer in the presence of DV3UTR3'siRNA2 and DV3UTR5'siRNA1. DENV3 RNA, quantified by qPCR, DV3UTR3'siRNA2 showed $80 \%$ reduction in DENV3 RNA level in comparsion with positive control cells having higher titers of DENV3. Finally, a negligible level of DENV3 E protein was detected in the supernatant of Vero-81 cells containing DV3UTR3'siRNA2. These findings suggest that DV3UTR3'siRNA2 and DV3UTR5'siRNA1 can significantly inhibit DENV3 in mammalian cell line.

Conclusion: Overall, the results demonstrate that DV3UTR3'siRNA2 and DV3UTR5'siRNA1 can become a potential vital component of a therapeutic formulation for major anti-dengue therapy against DENV3.
\end{abstract}

Keywords: Dengue virus, siRNA, anti-E antibody, Conserve regions, Vero-81 cells

Tropical Journal of Pharmaceutical Research is indexed by Science Citation Index (SciSearch), Scopus, International Pharmaceutical Abstract, Chemical Abstracts, Embase, Index Copernicus, EBSCO, African Index Medicus, JournalSeek, Journal Citation Reports/Science Edition, Directory of Open Access Journals (DOAJ), African Journal Online, Bioline International, Open-J-Gate and Pharmacy Abstracts

\section{INTRODUCTION}

Dengue virus (DENV) belongs to the genus Flavivirus and are named arboviruses, denoting their transmission via mosquitos. DENV transmission involves two vectors Aedes aegypti and Aedes albopictus. As per the recent valuation, approximately 50 to 100 million people are infected with DENV annually and 0.5 million progress to DHF/DSS [1]. DENV has a strand of positive sense RNA with the genome size of $11 \mathrm{~kb}$. DENV genome encodes a poly-protein, 
which is later cleaved into ten proteins [2]. DENV has four distinct serotypes (DENV1-4), which are genetically similar yet diverse on the basis of antigenic variation. DENV serotypes are further divided into genotypes on the basis of $3 \%$ variations within individual serotype [3].

Amongst many antiviral approaches, recent studies show that several RNA viruses are inhibited via RNA interference (RNAi) by targeting naked viral RNA [4]. RNA interference (RNAi) is a mechanism by which a certain RNA is degraded through host cellular machinery. Experimental data from various studies comprising of plasmids, synthetic inhibitors, and RNAi have shown promising results against Japanese Encephalitis virus (JEV), Dengue virus (DENV) and West Nile virus (WNV) $[5,6]$. In one study, genetically engineered mosquitoes were able to control DENV via RNAi where RNA interference targeted DENV RNA inside the host cell, reducing the copies of viral RNA and decreasing the overall viral load [7]. While targeting the conserved region of Envelope $(E)$ gene in the genome of DENV 2, it was observed that this siRNA can limit DENV 2 replication in human monocytes and macrophages [8]. Synthetic siRNA is usually 19 to 21 bps in length with 3 ' overhangs of two nucleotides. Highly conserved sequences were later used for siRNA design via different algorithms and then in experiments to observe anti-dengue potential in cell culture [9].

The objective of this study was to target conserve sequences in DENV3 genome by introducing siRNAs (small RNA molecules) which, in turn, will chop target regions via cellular machinery. By interfering with DENV3, genome viral replication can be blocked in mammalian cell line.

\section{EXPERIMENTAL}

\section{Viruses and cell lines}

On day 1 , Vero cells were grown in a growth medium comprising Dulbecco Eagle's medium (DMEM; Cellgro), $10 \%$ heat-inactivated fetal bovine serum (FBS), and panstrep (100 units/ml penicillin and $100 \mathrm{~g} / \mathrm{ml}$ streptomycin). Vero-81 cells were incubated overnight at $37{ }^{\circ} \mathrm{C}$ in a humid atmosphere while $5 \% \mathrm{CO}_{2}$ was maintained. DENV-3 (strain H87; GenBank accession no. M93130) were obtained from $\mathrm{Dr}$ Desilva's laboratory in Department of Microbiology and Immunology, University of North Carolina, Chapel Hill, USA.

\section{Bioinformatics for siRNA design}

DENV3 targeting siRNAs were designed by using Dharmacon algorithm (Dharmacon siRNA Design Center), which created optimized siRNAs to be used in anti-dengue assays $[9,10]$. Dharmacon algorithm generated several sequences on the bases of their likelihood to produce effective siRNAs (data not shown). Six non overlapping sequences were chosen from the top of the scoring list. For designing an siRNA that targets a highly conserved sequence in the DENV virome (conserved siRNA), alignment of complete genomes of reference strains were performed via TCoffee online software (http://www.tcoffee.org). Candidate sequences were aligned with the genomic library by using blastn program. Positive-control siRNA sequence from plant source was also prepared, which was not related to DENV, as shown later in Table 1. Conclusively, to minimize unplanned hybridization, all seven siRNAs were further checked by blastn program against human genomic sequences.

\section{Transfection (siRNA) of cell culture}

Transfection for performing cell culture experiments was done by applying reverse transfection in 48-well plates. In each of the experiment, suitable amount of 10 uM siRNA from the available stock (Dharmacon Inc, Lafayette, CO) was mixed with 1 ul of RNAiMAX (Invitrogen) which was later added to $100 \mathrm{ul}$ of Opti-DMEM for $30 \mathrm{~min}$ at room temperature [11]. Later, 100 ul of DMEM (with $2 \%$ FBS and no antibiotics) containing $2 \times 10^{5}$ Vero81 cells per well were added and gently mixed. The following day, the medium containing transfection mixture was removed, and virus diluted in 200 ul DMEM (containing $2 \%$ FBS and antibiotics) was used for infecting Vero-81 cells to produce 50 to 70 foci per well in 48 well plate that was kept in $\mathrm{CO}_{2}$ incubator for $2 \mathrm{~h}$ with constant shaking. After two hours of incubation, the medium (containing DENV) was removed and cells were washed twice with Opti-DMEM to completely remove unbound DENV particles concluding with adding 200 ul DMEM (containing $2 \%$ FBS and antibiotics) in each well. Supernatants were collected 48 hours post-infection (p.i.) and FRNT analysis was performed with remaining Vero 81 cells in each well.

\section{Focus assay}

Transfected cells (siRNAs) in 48 well plates were infected with 100 ul of diluted DENV3 to obtain 40 to 50 foci per well. A diluent, DMEM (100 ul), was added to each well to completely cover the 
surface area. After $1 \mathrm{~h}$ of incubation on a rocker, at $37^{\circ} \mathrm{C}$, cells were overlaid with $1 \mathrm{ml} 1.0 \%$ methylcellulose in OptiMEM (Gibco), supplemented with $2 \%$ FBS (Cellgro) and antibiotic mix (Gibco Antibiotic-Antimycotic). On the 3rd day of incubation $\left(37{ }^{\circ} \mathrm{C}, 5 \% \mathrm{CO}_{2}\right)$, methylcellulose containing overlay medium was removed. Subsequently, washing was performed using PBS, and cells were fixed in $80 \%$ methanol / 1x PBS and left to air-dry. For virus foci visualization, cells were blocked with blocking buffer (5\% instant milk in PBS), followed by incubation with anti-flavivirus MAb 4G2 that was diluted $1: 60$ in blocking buffer for $1 \mathrm{~h}$ at $37^{\circ} \mathrm{C}$, on a rocker. PBS was used for washing the wells after which cells were incubated with horseradish peroxidase (HRP), conjugated goat anti-mouse Ab (Sigma), diluted $1: 500$ in blocking buffer for $1 \mathrm{~h}$ at $37^{\circ} \mathrm{C}$, on a rocker. Before the addition of $150 \mathrm{ul} / \mathrm{well}$ of TrueBlue HRP substrate (KPL), plates were given a final washing. Lastly, viral foci were counted on a light box, enabling the calculation of viral titer by established methods.

\section{Real time PCR}

RNA copies were checked by real-time quantative polymerase chain reaction (RT-qPCR ) and quantification was carried out in CFX96TM PCR detection system using the iScriptTM RTPCR kit with SYBRH Green, as shown previously by Alhoot et al [12]. A total of three replicates were carried out for each sample along with a template control (negative control).

\section{Western blot analysis}

Samples were subjected to electrophoresis in Bio-Rad Mini protean 3, on a $12 \%$ separation gel. $20 \mathrm{ul}$ of samples were loaded to wells of the gel and ran at 100 Volts as per the protocol. After that, the gel was transferred to nitrocellulose membrane using semi-dry method (45 $\mathrm{V}$ with $0.35 \mathrm{~A}$ ). After transfer of the gel, the membrane was washed with washing buffer Tris buffer saline Tween 20 (TBST with $0.2 \%$ Tween20) and later blocked in the blocking buffer (2\% skim milk in TBST $0.05 \%$ Tween). Following that, the membrane was stained with primary antibody $8 \mathrm{~A} 1$ (1:1000 dilution of $10 \mathrm{mg} / \mathrm{ml} 8 \mathrm{~A} 1$ stock). Secondary staining was performed with goat anti-mouse HRP conjugated secondary antibody. Membranes were washed again 3 times and finally substrate was added to develop results on $x$-ray films.

\section{Immuno florescence assay (IFA)}

Micro-centrifuge tubes were labeled for each well, and cells were scrapped within an area of $1.5 \times 1.5 \mathrm{~cm}$. $1 \mathrm{~mL}$ of supernatant was used to briefly wash the scraped area, after which cells were pelleted in the micro-centrifuge tubes for 3 minutes, at $2500 \times \mathrm{g}$. A $10 \mathrm{uL}$ spot of resuspended pellet was made on each well, with even spreading, without scraping the poly-Llysine treated surface (coated with $0.0005 \%$ poly-L-lysine diluted in 1X PBS). The slides were air dried in the hood and incubated on ice for 10 minutes. For fixing cells, each slide was immersed in a $50 \mathrm{~mL}$ conical tube with $100 \%$ Acetone. Slides were dried and $25 \mathrm{uL}$ Block buffer $(5 \%$ Normal Mouse Serum diluted in 1X PBS) was added to each well and incubated for 30 minutes at room temperature. $2 \mathrm{H} 2-488$ antibody was used for pre staining diluted 1:200 in Block solution. Staining was performed by adding 25 ul stain solution to each well and incubated for 30 minutes in dark. After decanting, $35 \mathrm{uL}$ of $1 \mathrm{X}$ PBS was added and incubated in the dark for 5 minutes. Lastly, one drop of mounting fluid (Polysciences Inc., Aqua Poly/Mount 18606) was added to each well with application of cover slip. Each well of slide (Corning $22 \times 50 \mathrm{~mm}$ covers all ten wells) was observed under a fluorescent microscope.

\section{Statistical analysis}

Statistical analysis regarding siRNA inhibition effectiveness was performed by applying Kruskal-Wallis test using SPSS (SPSS version 17.0 software). Kruskal-W test is a nonparametric test, which involves group analysis of variances that can be implemented to compare scores on continuous variable for a number of groups usually above three. Based on this test, significance is based on $p$-values. If $p$ is less than $0.05(p<0.05)$, it indicates a significant difference in the groups in the continuous variable, as shown later in Table 2.

\section{RESULTS}

\section{Effective siRNA designing and synthesis}

More than 20 siRNA sequences were designed from three different regions of DENV3 strain (gene bank accession number JX669500), two each from 5'UTR, 3'UTR and structural regions, respectively. After further evaluation using different algorithms, six siRNA sequences were selected. After checking all the parameters, the following six siRNA sequences were synthesized for inhibition studies in Vero-81 cells, as shown in Table 1. 


\section{Focus data}

Focus reduction neutralization assay was performed for observing reduction in viral titer of DENV3. Vero-81 cells that were transfected with siRNA were challenged with DENV3. Dilutions of DENV3 were prepared to produce $40-60$ foci per well in a 48 well plate. Positive control wells had Vero-81 cells transfected with control siRNA that was not related with DENV, while the negative control well was without any virus infection.
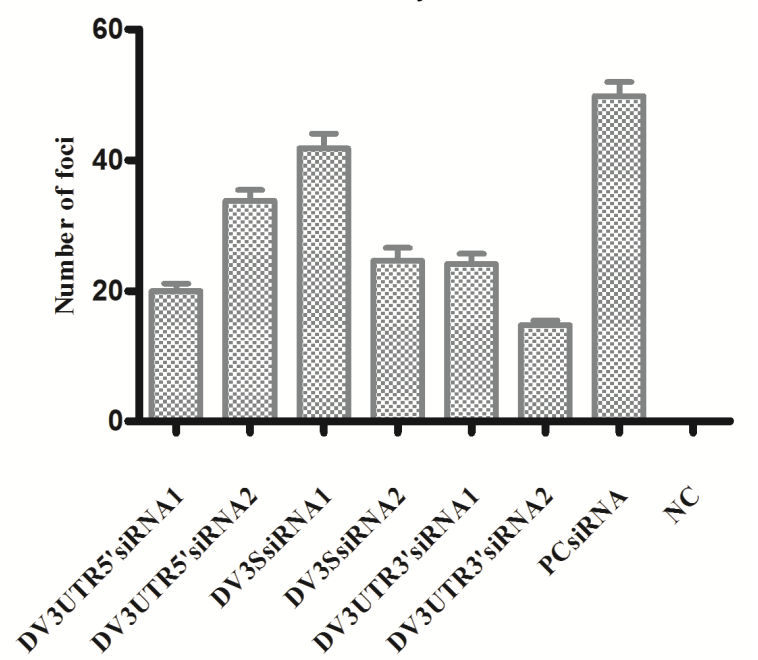

Fig 1: Foci count after $48 \mathrm{~h}$ of infection. Vero81 cells transfected with siRNAs designed against DENV3 were later challenged by DENV3 dilution (enough to produce 40 to 60 foci). Bar 1-6 siRNAs targeting DENV3 conserve sequences, bar 7 and 8 are positive control siRNA and negative control respectively. Experiments were repeated twice and similar results were obtained, reducing the likelihood of errors

On the third day post infection, FRNT assay was performed, in which DV3UTR3'siRNA2 was able to reduce virus titer up to $80 \%$, and siRNA designed from 5'UTR (DV3UTR5'siRNA1) showed marked decrease in DENV3 titer. Structural genes of DENV3 were also targeted by two siRNA sequences. DV3SsiRNA1 from structural region was unable to inhibit DENV3 as efficiently as DV3SsiRNA2 (Fig 1). These results demonstrate that siRNA based inhibition can effectively inhibit DENV3 in Vero-81.

\section{Immunoflorescence}

Immuno florescence assay was performed to check the level of inhibition achieved by siRNAs against DENV3. Vero-81 cells transfected with synthetic siRNAs were infected with DENV3, followed by performing IFA after 48 hours. Cells for individual wells were scrapped and visualized under a florescent microscope (Fig 2). IFA revealed that DV3SsiRNA2 had the maximum inhibition efficiency against DENV3 in Vero-81 cells. siRNAs designed from the structural regions were less effective with images showing less florescence as compared with siRNAs designed from UTRs. These observations suggest that DV3SsiRNA2 and DV3UTR5'siRNA1 were able to achieve approximately 70 to $85 \%$ reduction in viral titers.

\section{Real time PCR for quantification of DENV3 RNA}

Results obtained from Focus assay and IFA were later confirmed with real time qPCR analysis, and DENV3 RNA level was checked by using qPCR. A three-fold difference in DENV3 RNA was observed with DV3UTR3'siRNA2. DV3UTR3'siRNA1 also reduced DENV3 RNA levels, but this decrease in siRNA level was less than the one exhibited by DV3UTR3'siRNA2. While siRNAs designed against the structural region were not able to significantly reduce DENV3 RNA levels in infected Vero-81 cells, DV3SsiRNA2 was on the other hand, able to reduce DENV3 RNA up to $40-50 \%$ (Fig 3). Synthetic siRNA designed from 5'UTR were also highly effective in limiting DENV3 in Vero-81 cells, where a $50 \%$ reduction in DENV3 RNA with DV3UTR5'siRNA1 was observed.

\section{Western blotting}

Western blot analysis revealed that siRNAs targeting the $3^{\prime}$ and $5^{\prime}$ UTR of DENV3 were highly efficient in reducing viral titers in Vero-81 cells. Western blot was done using $8 \mathrm{~A} 1$ antibody targeting the $\mathrm{E}$ protein of DENV3. siRNA

Table1: Location of siRNA sequences in DENV 3 genome

\begin{tabular}{lcc}
\hline siRNA & Sequence (5'-3') & $\begin{array}{c}\text { Positions of siRNA sequence in } \\
\text { viral genome }\end{array}$ \\
\hline DV3UTR5'siRNA1 & UGCUUAACGUAGUGCUGAC & 49 \\
DV3UTR5'siRNA2 & CUUGCUUAACGUAGUGCUG & 27 \\
DV3SsiRNA1 & CGACAUGGGCUAUUGGAUA & 3001 \\
DV3SsiRNA2 & ACACCUGGACAGAGCAAUA & 2475 \\
DV3UTR3'siRNA1 & GACUAGAGGUUAGAGGAGA & 10566 \\
DV3UTR3'siRNA2 & GUGUAGCAGACUAGCGGUU & 10473 \\
PCsiRNA & ACGUGACGUUCGGAGAAUU & Plant source (control siRNA) \\
\hline
\end{tabular}




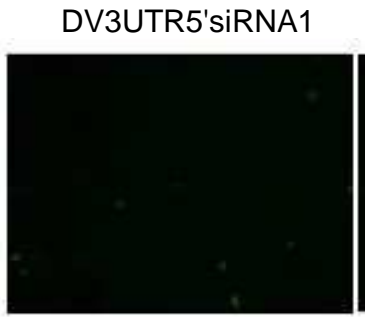

(a)

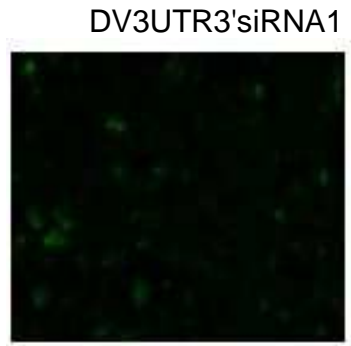

(e)

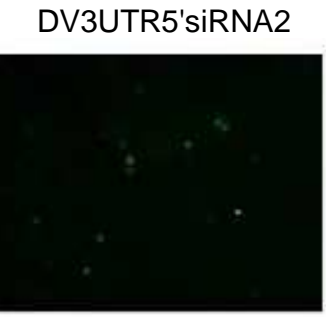

(b)

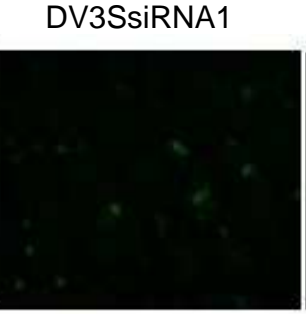

(c)
DV3SsiRNA2

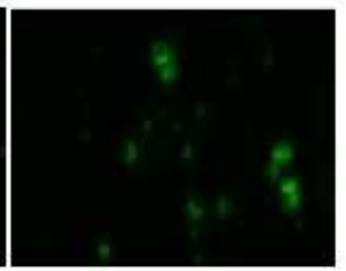

(d)

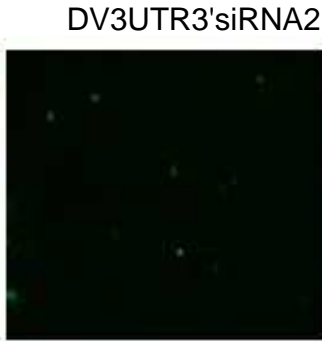

(f)

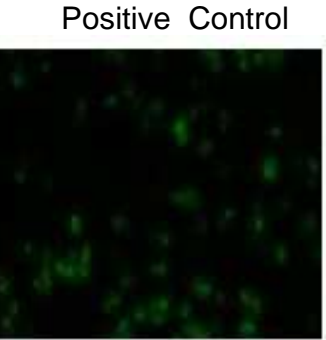

(g)
Negative Control

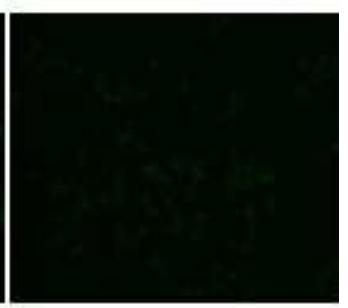

(h)

Fig 2: Vero-81 cells transfected with siRNAs stained by anti-E antibody (2H2-488), 48 h post-DENV3 infection. Images (a) and (b) are cells transfected with siRNAs, designed from the 5'UTR, showing marked reduction in viral load. Images (c) and (d) are Vero-81 cells transfected with siRNAs from structural region of the genome. A 30 to $40 \%$ reduction in viral load can be seen in the images. Images (e) and (f) display siRNAs that were designed from the 3'UTR of DENV3. Lastly, images $(\mathbf{g})$ and $(\mathbf{f})$ are positive control having PCsiRNA and no PCsiRNA, respectively

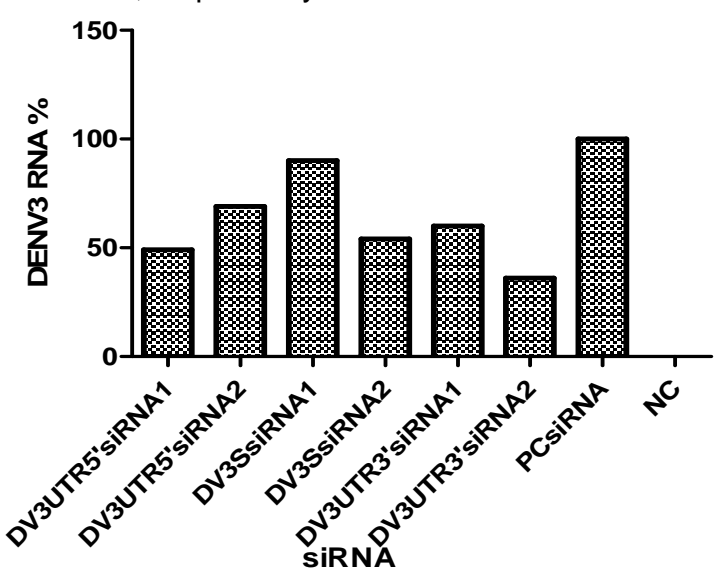

Fig 3: Real time qPCR performed 48 hours post infection. Bar 1 and 2 depict a decrease in DENV3 RNA by siRNAs targeting 5'UTR of DENV3. Bar 3 and 4 exhibit an effect of siRNAs designed from structural region of DENV3 genome. Bars 5 and 6 represent the levels of DENV3 RNA when treated with siRNAs against 3'UTR. Lastly, bars 7 and 8 are positive control containing PCsiRNA not related with DENV3 genome and negative control without any virus designed from structural regions had no significant effect on virus titer. Samples transfected with siRNAs designed from the structural regions had a higher band visibility.

\section{Statistical data}

The focus assay counts were later analyzed statistically using SPSS version 17. Standard deviation and $p$-value were obtained. The $p$ value of each SiRNA in comparison with the control was $\leq 0.05$, indicating that the variable is statistically significant and the siRNAs were highly effective.

\section{DISCUSSION}

With the recent advancements in the field of molecular biology, siRNA technology has become a leading tool in gene therapy with

Table 2: Statistical data for focus assay counts

\begin{tabular}{cccc}
\hline siRNA & Control & Counts (mean \pm SD) & P-value \\
\hline DV3UTR5'siRNA1 & PCsiRNA & $20 \pm 2.82$ & $>0.05$ \\
DV3UTR5'siRNA2 & PCsiRNA & $33.8 \pm 3.9$ & $>0.05$ \\
DV3SsiRNA1 & PCsiRNA & $41.8 \pm 5.4$ & $>0.05$ \\
DV3SsiRNA2 & PCsiRNA & $24.6 \pm 4.7$ & $>0.05$ \\
DV3UTR3'siRNA1 & PCsiRNA & $24.1 \pm 3.8$ & $>0.05$ \\
DV3UTR3'siRNA2 & PCsiRNA & $14.8 \pm 1.7$ & $>0.05$ \\
\hline
\end{tabular}


(a)

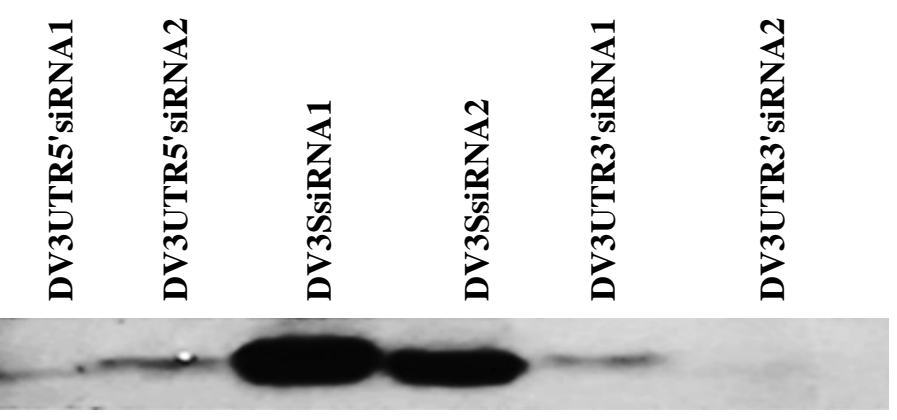

(b)

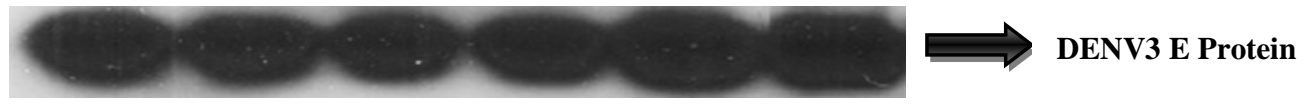

Fig 4: Western blot analysis of samples treated with six synthetic siRNAs. (a) Lane 1 and 2 represent siRNAs designed from the 5'UTR of DENV3. Lanes 3 and 4 show samples treated with siRNAs, targeting the structural region. Lane 5 and 6 are representative of samples treated with siRNAs from 3'UTR, respectively; (b) 1 to 6 represent control, DENV3 E protein

applications such as up-regulation and downregulation of certain target genes. Based on this enormous potential, siRNA technology is being extensively used for anti-virus drug development. With advantages like specificity, minimum or non-toxicity and efficient mode of action, siRNAs are one of the important components of biopharmaceuticals. Recent studies suggest that siRNAs can be used for blocking virus transmission [12,13]. DENV carrying a single stranded RNA as the genome in the cytoplasm is a probable target for different RNAi based inhibition mechanisms.

In the current study, we observed that synthetic siRNAs that targets the highly conserved regions of DENV3 genome can effectively inhibit DENV3 in Vero-81 cell lines. Reduction in viral loads were observed by immunoflorescence assay (IFA) and focus assay (FRNT), which were later confirmed by quantitative PCR (qPCR) and lastly by western blot analysis. These conserved regions of DENV genome are potential targets for effective antiviral drug designing and targeting. Additionally, this information may possibly be beneficial for future studies involving genome based inhibition of DENV. Several recent studies suggest that 3 'UTR of Dengue virus is crucial for proper RNA replication [14,15]. Diego et al exhibited that there is a 100 -fold decrease in Dengue virus RNA synthesis when deletions were introduced in 3'UTR of Dengue virus [16]. siRNA (DV3UTR3'siRNA2) designed from the conserved 3'UTR region was able reduce DENV3 RNA significantly, demonstrating $80 \%$ reduction in DENV3 titers. The highly conserved 10 nucleotide stretch at the 5'UTR of all Dengue serotypes depicts a pivotal role in Dengue virus replication cycle. RNAi interference at this location can significantly reduce Dengue virus replication resulting in reduction of virus titers [17-19]. As expected, siRNAs designed from 5'UTR region were also successful in limiting the viral load, where a 2 to 3 folds reduction with siRNA (DV3UTR5'siRNA1) was observed. Ultimately, one of the siRNA from the structural region of DENV genome managed to reduce viral titers in the range of 40 to $45 \%$.

These results demonstrate that siRNAs can serve as an effective molecular biological tool for inhibiting DENV in mammalian cells. With proper delivery mechanism in place such as, nanoparticles in combination with lipids, these siRNAs can be used for in vivo studies [20]. Our data suggests that synthetic siRNAs, when introduced, can reduce Dengue virus titers in the cell cultures. Further studies can enhance the efficiency of these powerful anti-virals by reducing siRNA degradation and sustaining their required levels in the cells.

\section{CONCLUSION}

Despite siRNAs being effective for inhibiting virus in cell line, there are many drawbacks associated with siRNAs, including a complex mechanism for delivery, rapid degradation in the cells, inefficient accumulation in the target cells and tissues, and failure to cross various membranes. According to recent studies, lipid nanoparticle (LNP) is the most promising technology for sustained siRNA delivery and currently, it is a part of more than five clinical trials in various phases. A similar mechanism can be applied for deploying these siRNAs for future development of biopharmaceuticals against DENV and other viruses. 


\section{REFERENCES}

1. Alhoot MA, Wang SM, Sekaran SD. Inhibition of Dengue Virus Entry and Multiplication into Monocytes Using RNA Interference. PLoS Negl Trop Dis 2011; 5: e1410.

2. Alvarez DE, De Lella Ezcurra AL, Fucito S, Gamarnik AV. Role of RNA structures present at the 3'UTR of dengue virus on translation, RNA synthesis, and viral replication. Virology 2005; 339: 200-212.

3. Anthony KG, Bai F, Krishnan MN, Fikrig E, Koski RA. Effective siRNA targeting of the 3' untranslated region of the West Nile virus genome. Antiviral Res 2009; 82: 166-168.Bhatt S, Gething PW, Brady OJ, Messina JP, Farlow AW, Moyes CL, Drake JM, Brownstein JS, Hoen AG, Sankoh O, et al. The global distribution and burden of dengue. Nature 2013; 496 : 504-507.

4. Diego EA, Ana Laura De LE, Silvana F, Andrea VG. Role of RNA structures present at the 3'UTR of dengue virus on translation, RNA synthesis, and viral replication. Virology 339 2005; 200-212.

5. Elghonemy S, Davis WG, Brinton MA. The majority of the nucleotides in the top loop of the genomic $3 \mathrm{~V}$ terminal stem loopstructure are cis-acting in a West Nile virus infectious clone. Virology 2005; 331(2): 238-246.

6. Filomatori CV, Lodeiro MF, Alvarez DE, Samsa MM, Pietrasanta L, Gamarnik AV. A 5' RNA element promotes dengue virus RNA synthesis on a circular genome. Genes Dev 2006; 20: 2238-2249.

7. Holmes EC, Twiddy SS. The origin, emergence and evolutionary genetics of dengue virus. Infect Genet Evol 2003; 3:19-28.

8. Huang $D D$. The potential of RNA interference-based therapies for viral infections. Curr HIV/AIDS Rep 2008; 5: 33-39.

9. Khromykh AA, Kondratieva N, Sgro JY, Palmenberg A, Westaway EG. Significance in replication of the terminal nucleotides of the flavivirus genome. J Virol 2003; 77(19): 10623-10629.

10. Khromykh AA, Meka H, Guyatt KJ, Westaway EG. Essential role of cyclization sequences in flavivirus RNA replication. J Virol 2001; 75: 6719-6728.
11. Lopez-Fraga $M$, Wright $N$, Jimenez A. RNA interferencebased therapeutics: new strategies to fight infectious disease. Infect Disord Drug Targets 2008; 8(4): 262273.

12. Munoz-Jordan JL, Sanchez-Burgos GG, Laurent-Rolle M, Garcia-Sastre A. Inhibition of interferon signaling by dengue virus. Proc Natl Acad Sci USA 2003; 100: 14333-14338.

13. Ong SP, Chu JJ, Ng ML. Inhibition of West Nile virus replication in cells stably transfected with vectorbased shRNA expression system. Virus Res 2008; 135: 292-297.

14. Reynolds $A$, Leake $D$, Boese $Q$, Scaringe $S$, Marshall WS, Khvorova A. Rational siRNA design for RNA interference. Nat. Biotechnol 2004; 22: 326-330.

15. Rico-Hesse R. Molecular evolution and distribution of dengue viruses type 1 and 2 in nature. Virology 1990; 174: 479-493.

16. Sánchez-Vargas I, Travanty EA, Keene KM, Franz AW, Beaty BJ, Blair CD, Olson KE. RNA silencing, Arthropod-borne virus and mosquitoes. Virus Research 2004; 102: 65-74.

17. Subramanya S, Kim SS, Abraham S, Yao J, Kumar M, Kumar $P$, Haridas V, Lee SK, Shultz LD, Greiner $D$ et al. Targeted delivery of small interfering RNA to human dendritic cells to suppress dengue virus infection and associated proinflammatory cytokine production. J Virol 2010; 84: 2490-2501.

18. Tilgner M, Deas TS, Shi PY. The flavivirus-conserved pentanucleotide in the $3 \mathrm{~V}$ stem-loop of the West Nile virus genome requires a specific sequence and structure for RNA synthesis, but not for viral translation. Virology 2005; 331 (2): 375-386.

19. Villordo SM, Alvarez DE, Gamarnik AV. A balance between circular and linear forms of the dengue virus genome is crucial for viral replication. RNA 2011; 16: 2325-2335.

20. Yuen Yi C. Tam, Sam Chen, Pieter R Cullis. Advances in Lipid Nanoparticles for siRNA Delivery. Pharmaceutic 2013; 5(3): 498-507. 